\title{
Fluctuations in Intracellular Calcium Responses to Action Potentials in Single En Passage Presynaptic Boutons of Layer V Neurons in Neocortical Slices
}

\author{
Bruno G. Frenguelli ${ }^{1}$ and Roberto Malinow ${ }^{2}$ \\ Cold Spring Harbor Laboratory \\ Cold Spring Harbor, New York 11724
}

\begin{abstract}
The release of neurotransmitter from a nerve terminal on invasion by an action potential shows large trial-to-trial fluctuations. The factors contributing to this variability have not been elucidated clearly. Here, simultaneous patch-clamp and optical measurements from layer $V$ neocortical pyramidal neurons have been used to assess the extent to which the fluctuations in transmitter release may be caused by variability in calcium rise in presynaptic structures. Boutons on axon collaterals were visualized and increases in intracellular calcium, assessed by Fura-2, were observed in response to single action potentials. In some boutons, calcium responses showed trial-to-trial variability and occasional apparent failures despite the faithful conduction of the action potential. These results suggest that a factor contributing to the fluctuation in transmitter release may be the variability with which depolarization of a presynaptic bouton produces an increase in intrabouton calcium.
\end{abstract}

\section{Introduction}

Although a rise in intracellular calcium is an absolute requirement for neurotransmitter re-

\footnotetext{
${ }^{1}$ Present address: Neurosciences Institute, Department of Pharmacology and Clinical Pharmacology, University of Dundee, Ninewells Hospital and Medical School, Dundee, DD1 9SY, Scotland, UK.

${ }^{2}$ Corresponding author.
}

lease, it is not clear what mechanisms contribute to the trial-to-trial fluctuations observed in central and peripheral nervous systems (Katz 1969; Llinas et al. 1981; Mulkey and Zucker 1991). There is evidence that in some conditions (Lüscher et al. 1994a,b; Wall 1995) an action potential triggered at a cell body may occasionally fail to conduct down the axon, thereby producing fluctuations in transmission. Furthermore, the absence of docked or primed vesicles at release sites could contribute to fluctuations in release. An alternative possibility examined in this study is that the increase in calcium triggered by an action potential in a small presynaptic bouton ( $2-3 \mu \mathrm{m}$ diameter) may vary from trial to trial. Given the steep dependence [ $\sim$ 4th power (Dodge and Rahamimoff 1967; Augustine and Charlton 1986; Zucker et al. 1991)] of transmitter release on calcium concentrations, small fluctuations in increases in intrabouton calcium would be expected to produce large changes in release probability. Such a small modulation could contribute to some forms of activity-dependent plasticity. Part of the material presented here has appeared in abstract form (B.G. Frenguelli and R. Malinow, pers. comm.).

\section{Materials and Methods}

\section{ELECTROPHYSIOLOGY AND \\ FLUORESCENCE IMAGING}

Neocortical slices $(400 \mu \mathrm{m})$ were prepared on a Vibratome from 11- to 16-day-old rat pups by use of standard techniques. Whole-cell recordings were made under visual guidance from superficial ( $<3$ cell bodies deep) Layer $\mathrm{V}$ pyramidal neurons with pipettes (1.2-2 $\mathrm{M} \Omega$ ) filled with $100 \mathrm{~mm}$ potassium gluconate, $40 \mathrm{~mm}$ HEPES, $5 \mathrm{~mm} \mathrm{MgCl}_{2}$,

LEARNING \& MEMORY 3:150-159 (c) 1996 by Cold Spring Harbor Laboratory Press ISSN1072-0502/96 \$5.00

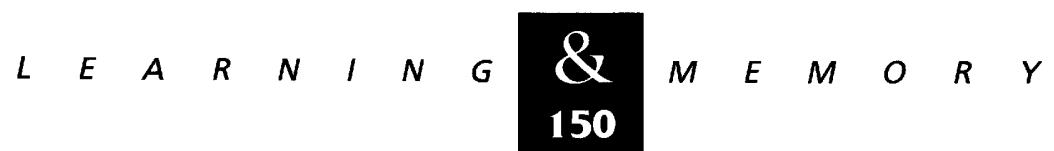


2 mм Na-ATP, $0.3 \mathrm{~mm}$ GTP, $5 \mathrm{~mm}$ glutathione brought to $\mathrm{pH} 7.3$ with $\mathrm{KOH}$. In addition, Fura-2 (Molecular Probes, Eugene, OR; Grynkiewicz et al. 1985) was added to the patch solution to a final concentration of $2 \mathrm{~mm}$. In some cells, biocytin $(0.2 \%)$ was added to the patch solution for subsequent immunohistochemistry. The oxygenated $\left(95 \% \mathrm{O}_{2} / 5 \% \mathrm{CO}_{2}\right)$ extracellular medium contained: $119 \mathrm{~mm} \mathrm{NaCl} ; 2.5 \mathrm{~mm} \mathrm{KCl} ; 1.3 \mathrm{~mm} \mathrm{MgCl}_{2}$; $1,2.5,7.5,10,15$, or $17.5 \mathrm{~mm} \mathrm{CaCl}_{2} ; 26.2 \mathrm{~mm}$ $\mathrm{NaHCO}_{3} ; 1 \mathrm{~mm} \mathrm{NaH} \mathrm{PO}_{4} ; 11 \mathrm{~mm}$ D-glucose at $\mathrm{pH}$ 7.4. Slices were placed within a submerged chamber at room temperature $\left(20-24^{\circ} \mathrm{C}\right)$ and individual pyramidal neurons viewed through an upright Zeiss Axioskop FS microscope equipped with a Zeiss Achroplan $40 \times, 0.75$ NA water immersion lens. Following diffusion of Fura- 2 into the patchclamped neuron, the main axon trunk was identified by its orientation relative to the plane of the slice, its smaller diameter relative to basal dendrites, and by the lack of spines that were clearly resolvable in basal and apical dendrites. Axon collaterals were seen under favorable recording conditions (recordings from surface cells with low series resistance; 3-10 M) after $\sim 10-15 \mathrm{~min}$ of Fura-2 dialysis. Boutons were identified on primary and secondary collaterals as small regions of increased fluorescence. No progressive changes in morphology (blebbing) were seen in any part of the neurons from which data was collected. We attribute this to the inclusion of the free-radical scavenger glutathione ( $5 \mathrm{~mm}$ ) in the patch solution.

Fluorescent illumination was provided by a $75 \mathrm{~W}$ xenon lamp and fluorescent images captured by a Photometrics cooled CCD camera in frame transfer mode. Experimental parameters (number of frames, frame interval, camera integration time, stimulus protocol) were specified by a Windowsbased custom imaging software package (WDI, Drs. Jay Callaway and William Ross) and executed by software written in Axobasic (Axon Instruments) on a separate PC (Gateway 2000). Synchronization of optical and electrophysiological acquisition was mediated by a Dagan LM-900 Laboratory Interface. The integration time for each CCD image was $150 \mathrm{msec}$; each pixel was 0.57 $\mu \mathrm{m}$; no pixel binning was done. Fluorescence images were collected at $380-\mathrm{nm}$ excitation. The change in fluorescence divided by the pre stimulus fluorescence, $\left(\mathrm{dF}_{\text {site }} / \mathrm{F}_{\text {site }}\right)$, was corrected for background autofluorescence (fluorescence in nearby region outside axon; $F_{\text {auto }}$ ); autofluores- cence correction factor $F_{\text {site }} /\left(F_{\text {site }}-F_{\text {auto }}\right)=\mathbf{4 . 1}$ \pm 2.8 (mean \pm S.D.) $n=44$. This correction factor was no different $(P>0.05)$ at sites showing trialto-trial variability compared with sites showing no variability. The resulting corrected $d F_{\text {site }} / F_{\text {site }}$ is a measure of a change in intracellular calcium. The value of $\mathrm{dF}_{\text {site }} / \mathrm{F}_{\text {site }}$ will saturate at higher $\mathrm{Ca}^{2+}$ levels $(1 \mu \mathrm{M})$. Although such saturation will tend to decrease the trial-to-trial variability we measure, we found no evidence for saturation during our test stimuli as repeated stimuli (e.g., 12 stimuli in $1.8 \mathrm{sec}$ ) produced considerably larger responses than single or double stimuli [ratio of mean response to repeated stimuli $(>2)$ to mean of double stimuli $=4.9 \pm 0.2 ; N=6]$.

\section{DOUBLE-LABELING IMMUNOHISTOCHEMISTRY}

In experiments in which cells had also been loaded with biocytin via the patch pipette, the pipette was gently removed and the slices were prepared for immunohistochemistry. Slices were fixed in $4 \%$ paraformaldehyde, $0.2 \%$ glutaraldehyde in $0.1 \mathrm{M}$ phosphate buffer (PB) for 12-14 hr at $4^{\circ} \mathrm{C}$ and equilibrated in $30 \%$ sucrose for $12-14$ hr. Cryosections $(30-\mu \mathrm{m})$ were permeabilized in $0.5 \%$ Triton-X and 5\% normal sheep serum (2-4 hr, $4^{\circ} \mathrm{C}$ ) and 1:200 Streptavidin Texas Red was added. After $24 \mathrm{hr}$ at $4^{\circ} \mathrm{C}$, sections were washed $(3 \times 10 \mathrm{~min})$ and incubated with or without primary antibody (monoclonal antisynatophysin, 1:50, Boehringer-Mannheim). Following wash ( $3 \times 10 \mathrm{~min}$ ), sections were incubated $\left(24 \mathrm{hr}, 4^{\circ} \mathrm{C}\right.$ ) in solution containing goat antimouse FITC (1:500). Sections were then washed $(3 \times 10 \mathrm{~min}$, $22^{\circ} \mathrm{C}$ ) and mounted in a 1,4-phenylenediamine (Aldrich) based fluorescence mounting medium ( $\mathrm{pH} \mathrm{8.0)}$ and stored at $-80^{\circ} \mathrm{C}$.

Digital epifluorescence images $(63 \times 0.9 \mathrm{NA}$, WI lens, Photometrics, PMIS) were obtained from sections. A section with a biocytin/Texas Red-labeled axon collateral was identified. Separate images displaying FITC and Texas Red fluorescence were obtained. A line was drawn through a region containing axonal varicosities in the Texas Red image and the corresponding line was drawn in the FITC image. The fluorescence intensities along these two lines, when plotted against one another, showed significant correlation (linear regression, $P<0.05$ for three separate experiments) indicating colocalization of synaptophysin to the bright regions indicated by Texas Red and similar to those

$$
\begin{array}{llllllllllllllll}
\hline & E & A & R & N & I & N & G & \mathbf{Q} & M & E & M & O & R & Y \\
151 & & & & &
\end{array}
$$


revealed by Fura 2 fluorescence. There was no significant correlation when the Texas Red intensity was compared with intensity profiles taken five pixels from the original line in the FITC image $(P>0.05, n=6)$. A concern was bleed-through of fluorescence from the Texas Red wavelength. We addressed this in a number of ways: We used a high power, high NA lens $(63 \times, 0.9 \mathrm{NA})$ to reduce fluorescence from out-of-focus regions; we used a narrow pass filter (35 nm; 535DF35; Omega Optical) to virtually $(<1 \%$ ) eliminate bleed-through from the Texas Red channel to the FITC channel; and we measured bleed-through directly by comparing digital images of a Texas Red-containing solution in the Texas Red and FITC channels. Bleed-through of images similar in absolute fluorescence intensity as those obtained for the tissue samples was typically $<1 \%$. By subtracting $1 \%$ of the Texas Red signal from the FITC image, a positive correlation of intensities was still significant $(n=3)$.

\section{DATA ANALYSIS}

Changes in fractional fluorescence $(\mathrm{dF} / \mathrm{F})$ at a varicosity were measured by choosing a box covering the site and calculating the difference between the average $\mathrm{dF} / \mathrm{F} 750 \mathrm{msec}$ (five frames) immediately before and immediately after an evoked action potential (bars in Fig. 2B, below). The difference of $\mathrm{dF} / \mathrm{F}$ between consecutive 750 msec periods obtained at the same sites in the absence of action potentials was used to calculate background noise; 13 such measurements were obtained from each baseline sweep (no elicited action potentials). Analysis was confined to regions of highest fluorescence precluding analysis of the axon between varicosities as these regions contained little fluorescence above the background. Sweeps were obtained approximately every 1-3 min. For analyzing amplitude distributions of responses to an action potential, sweeps contained one or two evoked action potentials (the latter evoked 1-1.5 sec apart). Sweeps with evoked action potentials were interleaved with baseline sweeps that were used for dye-bleaching correction and background noise measurements.

\section{CALCULATION OF SIGNAL VARIANCE}

Assuming that the signal and background noise vary independently, $\sigma_{T}^{2}=\sigma_{N}^{2}+\sigma_{S}^{2}$, where $\sigma_{T}^{2}$ is the variance measured among trials, $\sigma_{N}^{2}$ is the variance measured with no stimulus. Thus, the variance intrinsic to the signal, $\sigma_{\mathrm{S}}^{2}$, can be calculated. We used the F-test, which assumes a Gaussian distribution for the populations, to determine whether $\sigma_{\mathrm{T}}^{2}$ differs from $\sigma_{\mathrm{N}}^{2}$. The F-test compares the ratio of the two variances with the sample size used to generate them. In addition to the F-test conducted at each site, at 10 randomly chosen sites simulations were carried out to test the probability that $\sigma_{\mathrm{T}}^{2}$ was different from $\sigma_{\mathrm{N}}^{2}$. In these simulations, a set of $n$ values were randomly chosen from the set of noise measurements obtained at the site, where $n$ is the number of trial measurements. The variance was calculated for each of 1000 sets of $n$ randomly chosen values. The number of times that the variance of these $n$ values was equal to or exceeded the variance measured for the $n$ trials was denoted by $n(\mathrm{e})$. The probability that a variance equal to or larger than that measured for trials could come from a distribution like the noise is given by $n(e) / 1000$. For every case where the F-test indicated that $\sigma_{\mathrm{N}}^{2}$ was significantly different from $\sigma_{\mathrm{T}}^{2}$, these simulations also showed significance.

\section{ESTIMATION OF SIGNAL VARIANCE}

In 45 of 52 cases, the F-test indicated that $\sigma_{T}^{2}$ was not significantly greater than $\sigma_{\mathrm{N}}^{2}$. We estimated the predicted trial variance $\left(\mathbf{P \sigma}_{\mathrm{T}}\right)$ for the observed response mean $(M)$ had there been an average coefficient of variation $\left(\mathrm{CV}_{\mathrm{m}}\right)$ as seen at those sites that did show significant $\sigma_{\mathrm{T}}^{2} \cdot \mathbf{P \sigma}_{\mathrm{T}}^{2}=\sigma_{\mathrm{N}}^{2}$ $+\mathrm{M}^{2} \cdot \mathrm{CV}_{\mathrm{m}}^{2}$. In 19 of these 52 sites, an average amount of variance would not have been measured as significant by an F-test. This indicates that limitations in signal-to-noise resolution will tend to underestimate the fraction of sites that have a fluctuation in responses.

\section{Results}

Layer $V$ pyramidal neurons in $400-\mu \mathrm{m}$ neocortical slices were patch-clamped and dialyzed with a patch solution that contained the calcium indicator dye Fura-2 ( $2 \mathrm{~mm}$ ) (Grynkiewicz et al. 1985) and histological marker biocytin. Approximately $15 \mathrm{~min}$ after gaining whole-cell access, the axon and collaterals were identified. Primary and sec- 
ondary collaterals contained varicosities (regions of increased fluorescence) that showed little morphological change over the lifetime of the experiment ( $\sim 1$ hr on average; see Fig. $2 \mathrm{~A})$. The size of varicosities in the present study was estimated by measuring the distance from the brightest pixel to the pixel at which the intensity fell to $50 \%$ of that of the brightest. These structures, which measured $2.13 \pm 0.73$ by $1.71 \pm 0.66 \mu \mathrm{m}(n=22$; mean \pm s.D. $)$, resemble in size and morphology presynaptic boutons that have been identified with light and electron microscopy (Kisvárday et al 1986; Gabbott et al 1987; DeFelipe and Fariñas 1992; Deuchars et al. 1994).

To test if these varicosities were presynaptic boutons, the recording pipette was gently removed, and the slice was fixed and processed for double fluorescence labeling for biocytin and the synaptic vesicle protein synaptophysin. Digital fluorescence imaging of the biocytin label revealed clear structures corresponding to the varicosities seen in vivo with the Fura-2 fluorescence. Imaging of the synaptophysin label showed a punctate distribution of fluorescent signal (Fig. 1). Colocalization of biocytin and synaptophysin at varicosities was shown by measuring a positive correlation in intensities of the two fluorescent signals $(n=3$; Fig. 1). These results indicate that the varicosities observed with Fura- 2 imaging contained synaptic vesicle proteins and were, therefore, presynaptic boutons.

To examine evoked calcium entry in presynaptic boutons, whole-cell recordings were obtained with a solution containing Fura-2. Cells were briefly $(5-10 \mathrm{msec})$ depolarized $(+30$ to
$+60 \mathrm{mV}$ relative to rest) to generate an action potential and simultaneous optical measurements were made from boutons (Fig. 2B). A number of

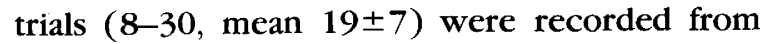
each varicosity ( 52 sites from 18 neurons). At all sites examined, the mean change in fluorescence produced by a single action potential was significantly different from the background noise $(P<0.025$, unpaired $t$-test) and was greater when extracellular calcium was raised. Responses to six spikes delivered at $3 \mathrm{~Hz}$ were measured in $2.5 \mathrm{~mm}$ $\left[\mathrm{Ca}^{2+}\right]_{0} ; \mathrm{dF} / \mathrm{F}=14.8 \pm 8.4 \% \quad$ (mean \pm s.D.; $n=10$ sites from three cells) and in $17.5 \mathrm{~mm}\left[\mathrm{Ca}^{2+}\right]_{0}$; $\mathrm{dF} / \mathrm{F}=23.4 \pm 7.7 \%(n=9$ sites from three cells $)$. These means are significantly different $(P<0.05$; unpaired $t$-test). Responses reached a peak within one image frame (150 $\mathrm{msec})$ and displayed a decay with a time constant of $3.7 \pm 1.5 \mathrm{sec}$ (mean \pm S.D.; $n=8$; Fig. 2C).

For each varicosity, we constructed an amplitude distribution histogram of the change in fluorescence produced by individual action potentials (trials; Fig. 3). Similar measurements, made in the absence of an action potential, generated an amplitude distribution of the background noise for each varicosity (noise, Fig. 3). In 7 of the 52 varicosities examined, the variance of responses during trials was significantly greater than the variance of the noise (F-test, see Materials and Methods). Varicosities showing variable responses were no different from varicosities showing no response variability with respect to (1) their general morphology, (2) their size, or (3) the surrounding boxes used in their analysis $(P>0.05$ for $(2)$ and (3); unpaired $t$-test). The significantly greater vari-
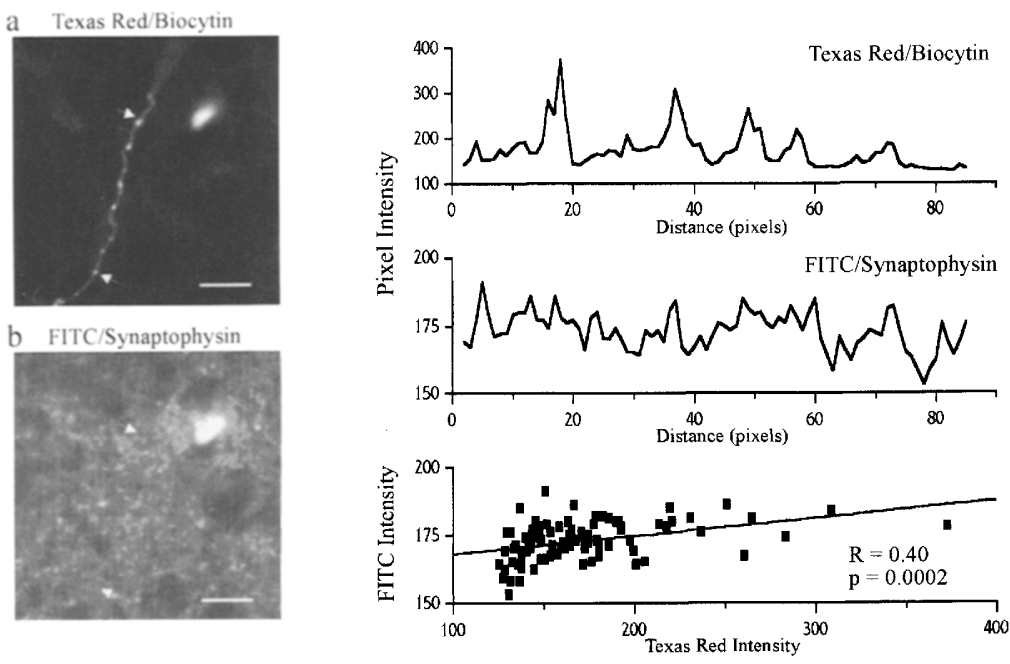

Figure 1: Colocalization of antisynaptophysin immunoreactivity $(b)$ to Texas Redlabeled axonal varicosities (a). A line was drawn between the arrowheads in a. The intensity profile along this line (left, upper graph) shows peaks corresponding to axonal varicosities in a. An identical line was drawn in the corresponding region in $b$. The intensity profile (left panel, middle graph) shows peaks corresponding to those in the upper (Texas Red/Biocytin) graph. A plot of Texas Red intensity vs. Fitc intensity (left panel, lower graph) shows a significant correlation between the two intensity profiles. Bars in a and $b, 10 \mu \mathrm{m}$. 
Figure 2: Single action potentials elicit increases in intracellular calcium in presynaptic boutons. (Aa) Fura-2 fluorescence image ( $380 \mathrm{~nm} ; 1$-sec exposure) of a Layer $V$ axon collateral in a 400- $\mu \mathrm{m}$ neocortical slice. Note small varicosities (putative presynaptic terminals) on the axon collateral. Region of high fluorescence in the upper right-hand corner originates from an out-of-focus basal dendrite. $(b, c)$ Enlarged fluorescence images of the secondary collateral in the boxed region in Aa taken 43 min apart showing the stability of the anatomical pattern. (c) Lower box contains the varicosity analyzed in $B$. Upper box defines the region from which the autofluores-
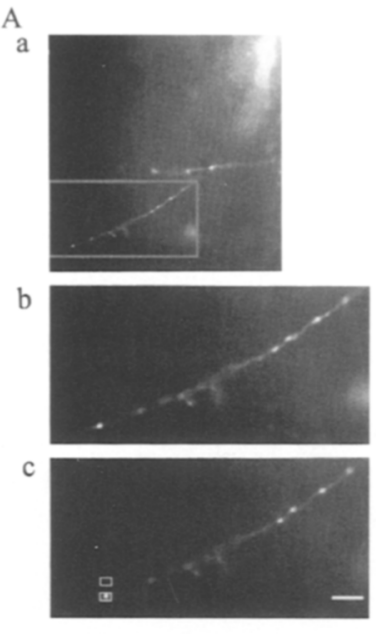
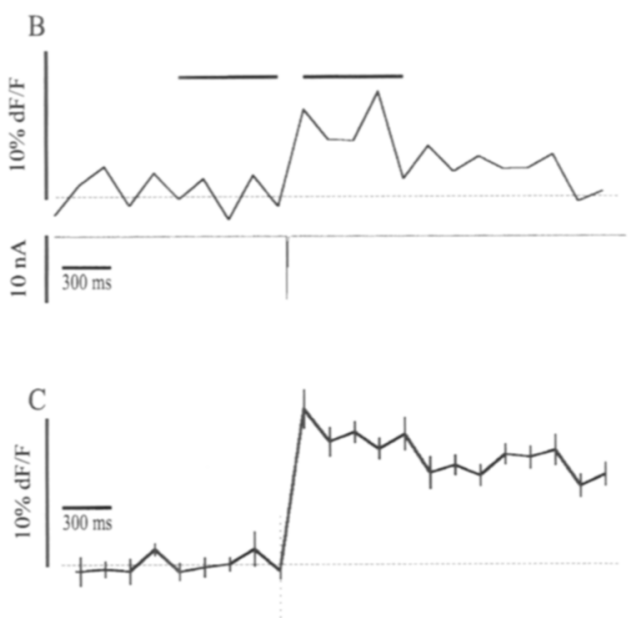

cence was measured. Bar, $10 \mu \mathrm{m}$. (B, top) Plot of $\mathrm{dF} / \mathrm{F}$ (corrected for autofluorescence and dye bleaching) vs. time for varicosity shown in AC. Each point indicates fluorescence integrated for $150 \mathrm{msec}$. Bottom, transmembrane current measured by patch electrode placed on the soma. Following a 1.5-sec baseline (10 images), a brief depolarizing step [5 $\mathrm{msec},+30 \mathrm{mV}$ from rest $(-60 \mathrm{mV})]$ was applied to the cell, via the patch-pipette. Note the fast sodium spike (bottom) and a triggered increase in calcium in the bouton (top). Bars above the trace indicate where amplitude measurements were made on each trial. Similar windows in the absence of stimuli measure background noise. (C) Average (mean \pm S.E.M.) of 11 responses to two action potentials at $50 \mathrm{~Hz}$ from a different bouton showing typical protracted time course of decay $(\tau=3.3 \mathrm{sec})$. Dotted vertical line indicates time of stimulation.

ance in responses suggests that the mechanisms responsible for generating the calcium signal have a measurable variability that cannot be accounted for by the variability in background noise.

As shown in Figure 3, the variability in responses relative to the mean could be quite large (Fig. 3Bb, sites 2 and 3), whereas for other sites (Fig. 3Ab, sites 1 and 2) little variability apart from the background noise could be detected. One possible mechanism that would produce a variable response at a site would be the probabilistic activation of a small number of calcium channels on a presynaptic bouton. If we assume that these channels act independently, then the mean and variance of the signal give an indication of the average number of channels acting in one trial and one can estimate the fraction of trials expected to produce no signal (failure of calcium to rise). Thus, if we assume $\mathrm{N}$ independent channels at each varicosity, each with probability $p$ of opening with an action potential, then the average number of channels opening on a trial $(\mathrm{m})$ is $\mathrm{m}=\mathrm{Np}<\mathrm{Np} /(1-\mathrm{p})$ $=$ mean $^{2} /$ variance.

For sites at which the variance of the response was significantly greater than the background noise, $\operatorname{mean}^{2} /$ variance $=3.2 \pm 0.7 \quad$ (mean \pm s.E.M., $\boldsymbol{n}=7$ ), suggesting that in this group of boutons a small number of channels act to produce a re- sponse. With such few channels acting, one would expect to see, on occasion, no channels opening in response to an action potential, with a resultant failure of calcium rise. Indeed, apparent failures in calcium rise at boutons were observed following the generation of an action potential at the cell body (e.g., Fig. 3Bb, site 3; Fig. 4).

Why is there a measurable variability in only a small subset of varicosities? Is this because only few boutons show variability or because of limitations imposed by the signal to noise resolution of our recording conditions? If we assume that all sites have the same variability (i.e., $\sigma /$ mean), we can ask: in how many of the sites which did not show a significant variability can this be attributed to a small mean response? That is, if the mean response is small, then the variability caused by the response will also be small. In this case, the variability could be dominated by the background variability (which should not depend on the mean response). We estimate (see Materials and Methods, "Estimation of Signal Variance") that of the 45 sites that did not show significantly greater variability than background, a variability comparable to that seen in sites showing variability would not be computed as significant in 19 sites, even though it exists. We conclude that in many sites there could be a variability that would not be observed

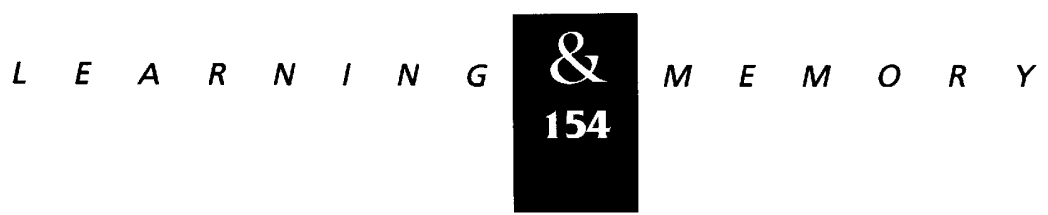


A a

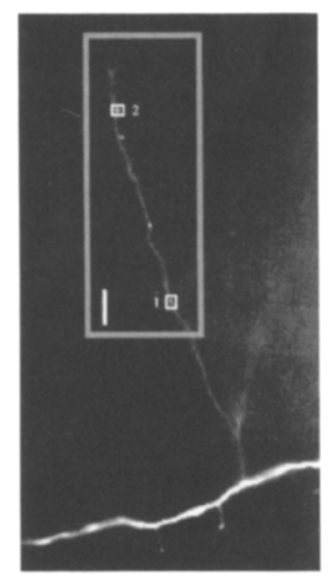

B a
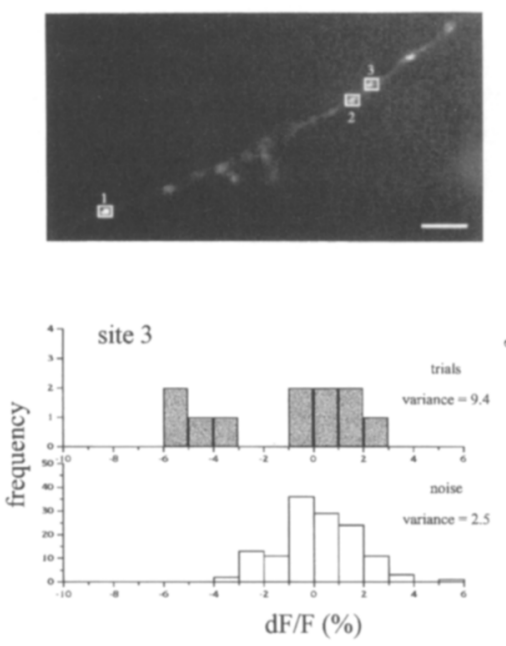

b

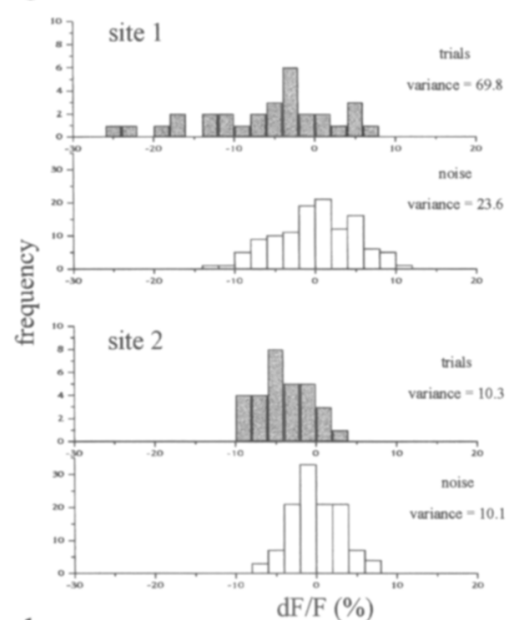

b

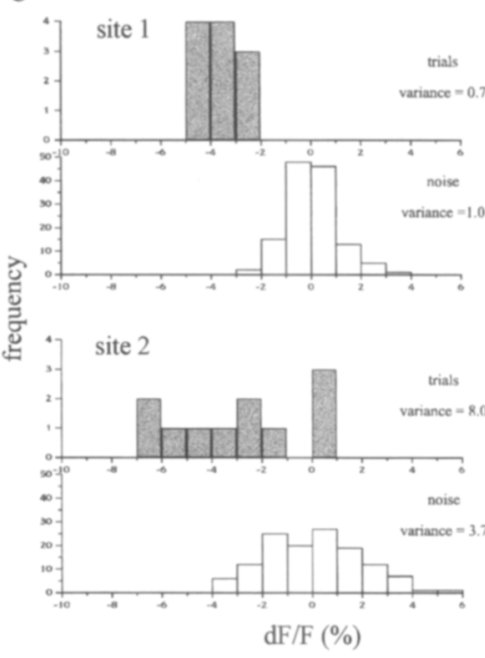

Figure 3: The calcium rise at varicosities evoked by single action potentials shows a variance that can be significantly greater than the variance of the background noise. (Aa, Ba) Fura-2 fluorescence images (380 $\mathrm{nm} ; A a, 1-\mathrm{sec}$ exposure; $B \mathrm{a}, 450-\mathrm{msec}$ exposure) of an axon and collaterals from two layer $\mathrm{V}$ neurons. Sites analyzed in $b$ are indicated. $(A b, B b)$ Amplitude distribution histograms for stimulus sweeps (trials) and baseline sweeps (noise) for different representative sites in the two neurons. Note the greater amplitude variability in trials compared with background noise in the sites Ab1 $(P<0.05 ;$ F-test), Bb2 $(P<0.05$; F-test), $(B b 3)(P<0.005 ; \mathrm{F}$-test) and the similar variability in trials as background noise in site Ab2 (P>0.05; F-test). (Bb1) One of four examples where the variability of the evoked trials was significantly less $(P<0.05 ;$ F-test $)$ than the variance of the background noise. (B) Same cell as Fig. 2; site 1 is more distal to the soma. Percent $\mathrm{dF} / \mathrm{F}$ values have been corrected for autofluorescence and dye bleaching. Bars in $A a$ and $B a, 10 \mu \mathrm{m}$. as significant because of the limitations of signal to noise resolution.

If calcium fails to rise at a varicosity when an action potential is elicited at the cell body, this could be because the action potential fails to reach a bouton. We have two pieces of evidence arguing against such a mechanism as being responsible for a significant amount of variability in the calcium responses. If the action potential were to fail to propagate, then one would expect that the rise of calcium at nearby varicosities would show a positive correlation among different trials. This is because these varicosities are arranged like beads on a string: If the action potential fails at one bead, all distal beads should also show failures. However, in none of the varicosities showing significant signal variance was the response amplitude for different trials significantly correlated with response amplitudes of other varicosities on the same branch
( $P>0.05$ for each pair, linear regression analysis). Furthermore, the independence of signal at different boutons is supported by the data shown in Figure 4, in which there are apparent failures of calcium increase at proximal sites with a simultaneous response at more distant sites. This indicates that a failure of the action potential to propagate cannot explain the apparent failure of calcium to increase.

We tested the hypothesis that the variability in calcium signal seen at some boutons was caused by the stochastic behavior of a small number of calcium channels. We reasoned that such boutons may have channels in a state of modulation that favors a closed state which can be overcome by prolonged (Bean 1989; Delcour and Tsien 1993; Delcour et al. 1993) or repeated (Fenwick et al. 1982; Hoshi et al. 1984) depolarization. In this case, repeated stimulation could maximally open

$$
\begin{array}{llllllllllllllll} 
& E & A & R & N & I & N & G & \begin{array}{l}
\boldsymbol{Q} \\
155
\end{array} & M & E & M & O & R & Y
\end{array}
$$



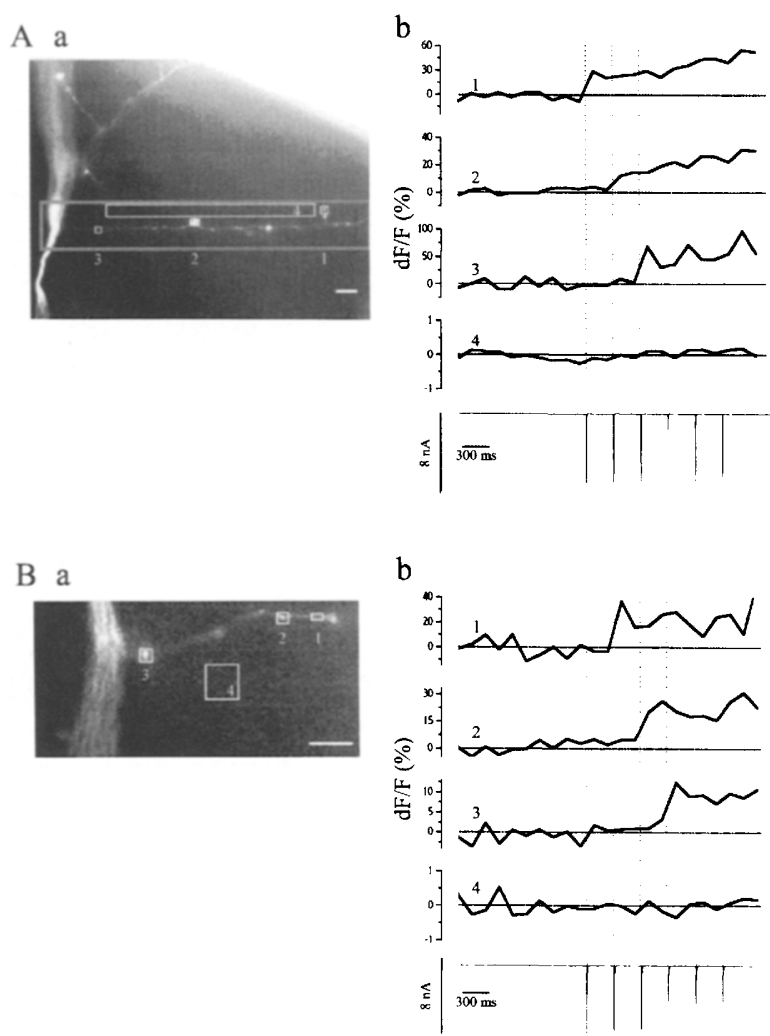

Figure 4: Apparent failures in calcium responses with faithful action potential propagation. ( $A a, B a$ ) Fura-2 fluorescence images (380-nm excitation; $A a, 500 \mathrm{msec}$ exposure; $\mathrm{Ba}, 450-\mathrm{msec}$ exposure) of two different axon collaterals filled with Fura- 2 from the same layer $V$ neuron showing multiple boutons. Numbers correspond to $\mathrm{dF} / \mathrm{F}$ vs. time plots in $A b$ and $B b$. $(A b, B b) A$ train of evoked action potentials (six spikes at $3 \mathrm{~Hz}$ ) elicits rises in different sites at different times. Note that an early spike elicits a response at distal sites whereas more proximal boutons fail to show increases in intracellular calcium until later spikes. These data argue against failure of action potential conduction as being responsible for the failure to show a calcium response. The failure order was in general random. Vertical dotted lines indicate time of stimulation. Background measurements indicate no generalized increases in fluorescence. $A b$ and $B b$ are separated in time by $47 \mathrm{~min}$. Bars, $10 \mu \mathrm{m}$.

channels, and thereby produce responses with little variability. Thus, we compared the responses to single stimuli and double stimuli (action potentials separated by $20 \mathrm{msec}$ ). We examined 42 sites in 14 neurons. Two stimuli produced a response that was on average $2.1 \pm 0.1$-fold (mean \pm S.E.M.; $n=42$ ) greater than a single trial, suggesting that there was little net facilitation of calcium entry. At these 42 sites, the responses to two stimuli showed no greater variance than responses to one stimulus (ratio $250 \mathrm{~Hz} / 21 \mathrm{~Hz}=1.0 \pm 0.2$; mean \pm S.E.M.; $n=42$ ). At the seven sites showing significant signal variance to one stimulus, the variance for double stimuli was, if anything, less than for one stimulus $(0.74 \pm 0.17$; Fig. 5$)$. The lack of increase in variance with two stimuli was not caused by saturation (a ceiling effect) because delivery of a larger number of stimuli produced larger responses (see Materials and Methods). These data suggest that the variance in the calcium increase can be modulated by repetitive activity.

We noted that in some sites ( 4 of 52), there was significantly more variability in the baseline periods compared with the response to single action potentials. We hypothesized that this may be caused by spontaneous calcium entry during baseline periods. We tested the possibility of spontaneous calcium entry by measuring the baseline noise of the optical signal at different external calcium concentrations. In four cells (13 sites), external calcium was increased from 1 or $2.5 \mathrm{~mm}$ to either 10 or $15 \mathrm{~mm}$. This increase in extracellular calcium resulted in a $1.97 \pm 0.52$-fold increase in variance (mean \pm S.E.M.; $n=13 ; P=0.056$, paired $t$-test). In 4 of these 13 sites, there was significantly more noise (in the absence of stimuli; $P<$ 0.05 ; F-test) when measured in high calcium compared with low calcium in the bathing medium. In addition, the three sites showing the greatest level of initial variance in low calcium all showed decreases in variance in high calcium. This supports the hypothesis that calcium channels can undergo a form of occlusion following their activation. Taken together, these data are consistent with the hypothesis that at some presynaptic sites there is spontaneous calcium channel activity.

\section{Discussion}

In this study, we have tested the hypothesis that the trial-to-trial fluctuation in transmitter release observed at central synapses may be caused by, at least in part, variable amounts of evoked calcium rise at presynaptic boutons. In agreement with previous results, we are able to measure a calcium signal in single presynaptic boutons evoked by an action potential (Regher et al. 1994). The origin of the calcium producing this signal could be from extracellular and/or intracellular pools. Two pieces of evidence argue against the latter possibility. First, the signal measured was smaller when extracellular calcium was lowered,

$$
\begin{array}{llllllll}
L & E & A & R & N & I & N & G \\
\begin{array}{llllllll}
\boldsymbol{Q} \\
156
\end{array} & M & E & M & O & R & Y
\end{array}
$$


A

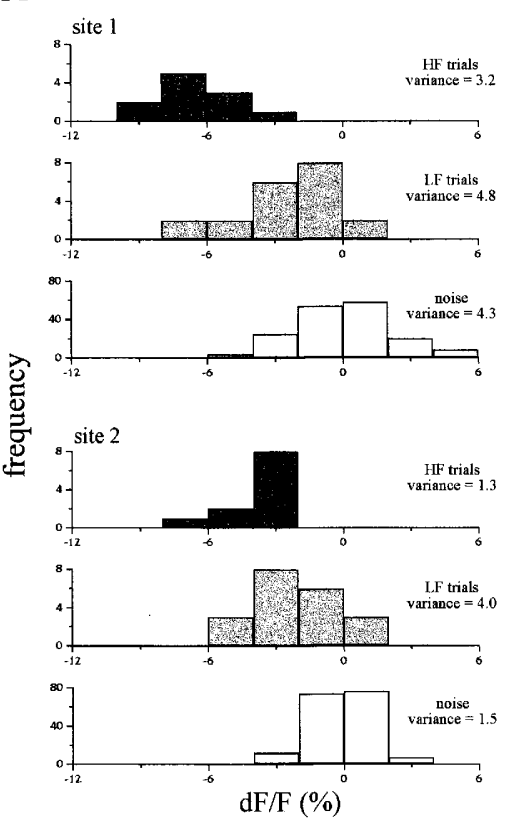

B
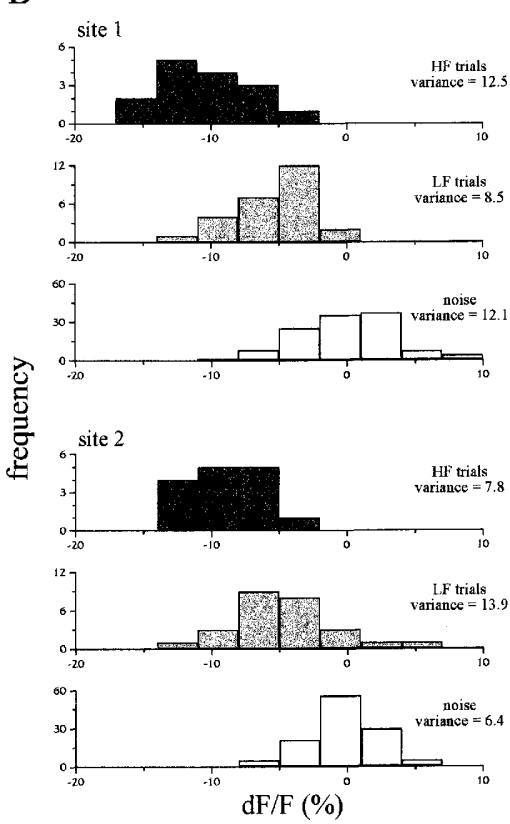

Figure 5: Modulation of response amplitude variance by repetitive activity. $(A, B)$ Two sites each from two different cells in which two shocks delivered at 50 $\mathrm{Hz}$ (HF trials, dark gray bars) caused no significant further increase in amplitude variance than that of 2 shocks at $1 \mathrm{~Hz}$ (LF trials, light gray bars). (A, site $1 ; B$, site 1 ) Variance of LF trials was not significantly greater than that of the noise. $(A$, site 2; $B$, site 2 ) Variance of LF trials was significantly greater than the variance of the noise. Note the pronounced reduction of variance following the $\mathrm{HF}$ trials.

suggesting that entry from the outside contributed appreciably to the evoked responses. Secondly, our data suggests that a relatively high Fura- 2 concentration reached boutons. This is indicated by the threefold slower decay of calcium signals in this study compared with another study using a lower concentration of Fura-2 (Regher et al. 1994). Thus, most of the calcium entering is likely to be well buffered (Neher and Augustine 1992) thereby minimizing calcium-induced calcium release from internal stores. Nevertheless, we have not ruled out that some of the calcium signal we observe may be caused by release of calcium from internal stores, which have been identified at axon terminals (Takei et al. 1992; Johnson et al. 1993).

By controlling the membrane potential of the soma connected to the boutons being examined, we are able to reliably elicit action potentials and thus ascribe variability in optical responses to factors other than stimulation variability (a complication with extracellular stimulation). We find that in $\sim 13 \%$ of the boutons the increase in calcium varies considerably from trial to trial. This variability could originate from a number of sources. For the reasons mentioned above, failure of action potential conduction seems an unlikely source. If the signal we observe were primarily from release from internal stores, then small fluctuations in calcium entry about a threshold value that triggers calcium release could magnify these fluctuations. Our results, however, can be most simply explained by the action of a small number of voltage-activated calcium channels with a low probability of opening during an action potential. The probability that a calcium channel opens during an action potential may be small if (1) the activation time is long, or (2) the modulated status of the channel favors a closed state (Bean 1989; Delcour and Tsien 1993; Delcour et al. 1993). The variance we observe for the signal could be caused by a variable number of calcium channels opening or variable duration of opening. The opening of a single calcium channel for one millisecond (the approximate duration of an action potential) can raise calcium concentration of a small $(2.5-\mu \mathrm{m}$ diameter) structure by $200 \mathrm{~nm}$ (Dunlap et al. 1995), levels that can readily be measured with Fura- 2 . Thus, assuming that the buffering at a bouton is dominated by the added Fura-2, influx through a small number of calcium channels could provide the signals we have observed. At most sites ( 45 of 52 sites), we observed no significant variability in the calcium signal. We estimate that in at least 19 of the 45 cases, we would not detect a significant variability (even though it exists) because the signal (and thus the variability) was too small (see Materials and Methods). In other cases, this is apparently not the reason for a consistent response (e.g., Fig. 3Bb, site 1) as responses were as large as those which showed variability. In these latter

$$
\begin{array}{lllllllllllllllll}
L & E & A & R & N & I & N & G & \bigotimes_{15} & M & E & M & O & R & Y
\end{array}
$$


cases, the calcium channels responsible for a consistent signal may be in a mode with a high probability of opening (Bean 1989; Delcour and Tsien 1993; Delcour et al. 1993).

We have compared the variance of responses to single or double stimuli. We find no increase in the variance of responses to double stimuli. We propose two possibilities to explain this observation. One possibility is that the variance to one stimulus is largely caused by background variance (even in the seven cases where it appears to be significantly different from background noise). In this case, one would not expect greater variance in response to two stimuli. An alternate possibility is that the responses to the second stimulus depends in some way on the response to the first stimulus. It could be that the variance we see is caused by stochastic opening of channels. In this case, channels in a low probability of opening (and thus high variance) could be converted to a high probability of opening (and, therefore, low variance) by repetitive depolarization. The net result would be that the variance to double stimuli would equal the variance to a single stimulus. If there is a reduction in response variance with double stimuli compared with a single stimulus (e.g., Fig. 5), then this suggests additional constraints. Such results suggest that a more consistent response is seen to two stimuli, than to a single stimulus. These data can be explained by a model in which (1) there is a small number of activatable channels; (2) the probability of a channel opening with a single action potential is small; (3) if a channel does not open with a single action potential, then the probability of opening with the second action potential is high (close to one); and (4) if a channel opens with the first action potential, the probability of opening with the second action potential is low (close to zero). This model is supported by a number of previously published observations. At a neuromuscular junction active zone there are a small number (15) of particles thought to be calcium channels (Pumplin et al. 1981; Walrond and Reese 1985). Calcium channels can be in a low probability of opening state (Bean 1989; Delcour and Tsien 1993; Delcour et al. 1993) that can be overcome by depolarization (Bean 1989; Fenwick et al. 1982; Hoshi et al. 1984) and calcium channels at release sites can be refractory to subsequent activation (Llinas et al. 1992). More complicated schemes may be necessary to incorporate the role of spontaneous calcium channel opening in the background noise we measure.
The calcium increases we have observed at presynaptic boutons display some similarities to neurotransmitter release. Both processes can show large fluctuations with intermittent failures. Furthermore, we find heterogeneity in the calcium response patterns; some sites show large variability while others do not. A similar heterogeneity in the probability of release has been described at central synapses (Hessler et al. 1993; Rosenmund et al. 1993; Thomson and Deuchars 1994). Activity patterns can modulate transmitter release and can result in various forms of synaptic plasticity (Zucker 1989). Here we find that the variability of responses may be affected by repetitive stimuli raising the possibility that under certain circumstances modulation of the probability of opening of calcium channels could underlie some forms of short- or long-term plasticity. In conclusion, our observations suggest that fluctuations in synaptic transmission may be caused by, at least in part, variable opening of calcium channels in small presynaptic boutons. However, a better signal to noise resolution may be necessary to conclude this more definitively.

\section{Acknowledgments}

B.G.F. is in receipt of a Wellcome Trust (UK) International Fellowship. We are grateful to Neal Hessler and Aneil Shirke for help with computer programming; Mirjana Savatic, David Spector, and Holly Cline lab for help with immunofluorescence; and Mike Silver and Michael Stryker for the immunofluorescence protocol.

The publication costs of this article were defrayed in part by payment of page charges. This article must therefore be hereby marked "advertisement" in accordance with 18 USC section 1734 solely to indicate this fact.

\section{References}

Augustine, G.J. and M.P. Charlton. 1986. Calcium dependence of presynaptic calcium current and post-synaptic response at the squid giant synapse. J. Physiol (London) 381: 619-640.

Bean, B.P. 1989. Neurotransmitter inhibition of neuronal calcium currents by changes in channel voltage dependence. Nature 340: 153-156.

DeFelipe, J. and I. Fariñas. 1992. The pyramidal neuron of the cerebral cortex: Morphological and chemical characteristics of the synaptic inputs. Prog. Neurobiol. 39: 563-607.

Delcour, A.H. and R.W. Tsien. 1993. Altered prevalence of gating modes in neurotransmitter inhibition of N-type calcium channels. Science 259: 980-984. 
Delcour, A.H., D. Lipscombe, and R.W. Tsien. 1993. Multiple modes of $\mathrm{N}$-type calcium channel activity distinguished by differences in gating kinetics. I. Neurosci. 13: $181-194$.

Deuchars, J., D.C. West, and A.M. Thomson. 1994. Relationships between morphology and physiology of pyramid-pyramid single axon connections in rat neocortex in vitro. J. Physiol. (Lond.) 478: 423-435.

Dodge, F.A.J.R. and R. Rahamimoff. 1967. Co-operative action of calcium ions in transmitter release at the neuromuscular junction. J. Physiol. (Lond.) 193: 419-432.

Dunlap, K., J.I. Luebke, and T. Turner. 1995. Exocytotic $\mathrm{Ca}^{2+}$ channels in mammalian central neurons. Trends Neurosci. 18: 89-98.

Fenwick, E.M., A. Marty, and E. Neher. 1982. Sodium and calcium channels in bovine chromaffin cells. J. Physiol. (London) 331: 599-635.

Gabbott, P.L.A., K.A.C Martin, and D. Whitteridge. 1987. Connections between pyramidal neurons in layer 5 of cat visual cortex (area 17). J. Comp. Neurol. 259: 364-381.

Grynkiewicz, G., M. Poenie, and R.Y. Tsien. 1985. A new generation of $\mathrm{Ca}^{2+}$ indicators with greatly improved fluorescence properties. J. Biol. Chem. 260: 3440-3450.

Hessler, N.A., A.M. Shirke, and R. Malinow. 1993. The probability of transmitter release at a mammalian central synapse. Nature 366: 569-572.

Hoshi, T., J. Rothlein, and S.J. Smith. 1984. Facilitation of $\mathrm{Ca}^{2+}$ channel currents in bovine adrenal chromaffin cells. Proc. Natl. Acad. Sci. 81: 5871-5875.

Johnson, R.J., H.Y. Pyun, J. Lytton, and R.E. Fine. 1993. Differences in the subcellular localization of calreticulin and organellar $\mathrm{Ca}^{2+}$-ATPase in neurons. Mol. Brain Res. 17: 9-16.

Katz, B. 1969. The release of neural transmitter substances: The Sherrington lectures X. Liverpool University Press, Liverpool, UK.

Kisvárday, Z.F., K.A.C. Martin, T.F. Freund, Z.S. Maglóczky, D. Whitteridge, and P. Somogyi. 1986. Synaptic targets of HRP-filled layer III pyramidal cells in the cat striate cortex. Exp. Brain Res. 64: 541-552.

Llinas, R., I.Z. Steinberg, and K. Walton. 1981. Relationship between presynaptic calcium current and postsynaptic potential in squid giant synapse. Biophys. J. 33: 323-351.

Llinas, R., M. Sugimori, and R.B. Silver. 1992.

Microdomains of high calcium concentration in a presynaptic terminal. Science 256: 677-679.

Lüscher, C., J. Streit, P. Lipp, and H.R. Lüscher. 1994a. Action potential propagation through embryonic dorsal root ganglion cells in culture. II. Decrease of conduction reliability during repetitive stimulation. I. Neurophysiol. 72: 634-643.

Lüscher, C., J. Streit, R. Quadroni, and H.R. Lüscher. 1994b. Action potential propagation through embryonic dorsal root ganglion cells in culture. I. Influence of the cell morphology on propagation properties. J. Neurophysiol. 72: 622-633.

Mulkey, R.M. and R.S. Zucker. 1991. Action potentials must admit calcium to evoke transmitter release. Nature 350: 153-155.

Neher, E. and G.J. Augustine. 1992. Calcium gradients and buffers in bovine chromaffin cells. ). Physiol. (London) 450: 273-301.

Pumplin, D.W., T.S. Reese and R. Llinas. 1981. Are the pre-synaptic membrane-particles the calcium channels. Proc. Natl. Acad. Sci. 78: 7210-7213.

Regehr, W.G., K.R. Delaney, and D.W. Tank. 1994. The role of presynaptic calcium in short-term enhancement at the hippocampal mossy fiber synapse. J. Neurosci. 14: 523-537.

Rosenmund, C., J.D. Clements, and G.L. Westbrook. 1993. Nonuniform probability of glutamate release at a hippocampal synapse. Science 262: 754-757.

Takei, K., H. Stukenbrok, A. Metcalf, G.A. Mignery, T.C. Sudhof, P. Volpe, and P. De, Camilli. 1992. $\mathrm{Ca}^{2+}$ stores in Purkinje neurons: Endoplasmic reticulum subcompartments demonstrated by the heterogeneous distribution of the InsP3 receptor, $\mathrm{Ca}^{2+}$-ATPase, and calsequestrin. J. Neurosci. 12: $489-505$.

Thomson, A.M. and J. Deuchars. 1994. Temporal and spatial properties of local circuits in neocortex. Trends Neurosci. 17: 119-126.

Wall, P.D. 1995. Do nerve impulses penetrate terminal arborizations? A pre-presynaptic control mechanism. Trends Neurosci. 18: 99-103.

Walrond, J.P. and T.S Reese. 1985. Structure of axon terminals and active zones at synapses on lizard twitch and tonic muscle fibers. J. Neurosci. 5: 1118-1131.

Zucker, R.S. 1989. Short-term synaptic plasticity. Annu. Rev. Neurosci. 12: 13-31.

Zucker, R.S., K.R. Delaney, R. Mulkey, and D.W. Tank. 1991. Presynaptic calcium in transmitter release and posttetanic potentiation. Ann. New York Acad. Sci. 635: 191-207.

Received June 5, 1996; accepted in revised form July 26, 1996.

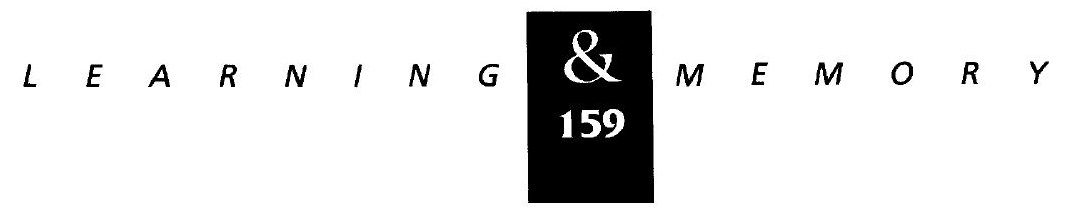




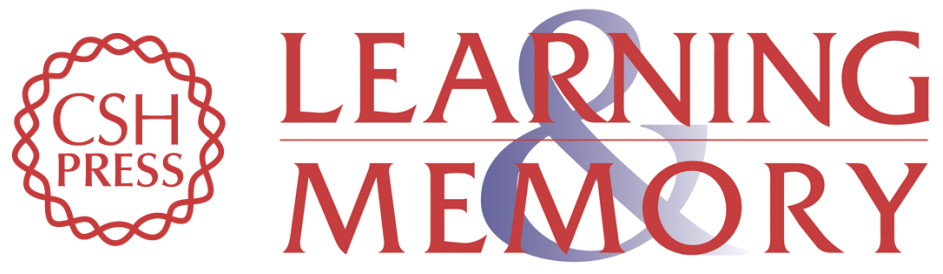

\section{Fluctuations in intracellular calcium responses to action potentials in single en passage presynaptic boutons of layer $\mathrm{V}$ neurons in} neocortical slices.

B G Frenguelli and R Malinow

Learn. Mem. 1996, 3:

References This article cites 30 articles, 10 of which can be accessed free at: http://learnmem.cshlp.org/content/3/2-3/150.full.html\#ref-list-1

License

Email Alerting Receive free email alerts when new articles cite this article - sign up in the box at the Service top right corner of the article or click here. 